\title{
Response to comment on 'Existing prognostic models, but not neutrophil-to-lymphocyte ratio, are prognostic in malignant mesothelioma'
}

\author{
T M Meniawy ${ }^{\star 1,2,3}$, J Creaney ${ }^{1,2}, \mathrm{R}^{\mathrm{A}}$ Lake $^{1,2}$ and A K Nowak ${ }^{1,2,3}$
}

${ }^{1}$ M503, School of Medicine and Pharmacology, University of Western Australia, Sir Charles Gairdner Hospital, Nedlands, Western Australia 6009, Australia; ${ }^{2}$ National Centre for Asbestos Related Disease, Sir Charles Gairdner Hospital, Nedlands, Western Australia 6009 , Australia and ${ }^{3}$ Department of Medical Oncology, Sir Charles Gairdner Hospital, Nedlands, Western Australia 6009, Australia

Sir,

Our goal when this study was designed was to understand whether the findings of the initial study (Kao et al, 2010) could also be useful at other clinical time points, and whether neutrophil-to-lymphocyte ratio (NLR) retained independent significance when established clinical prognostic factors were incorporated. The data did not support our initial hypothesis, and the correspondents take issue with this (Kao et al, 2014). The validation of prognostic markers in retrospective series frequently generates conflicting findings, and we all await a robustly designed prospective study to resolve this controversy.

The correspondents suggest that our study was flawed due to the inclusion criteria that they considered were arbitrarily defined and that led to selection bias. We acknowledge that this is an inherent limitation of all retrospective studies, including previous studies of the NLR. In the initial study (Kao et al, 2010), only patients receiving systemic therapy were selected, and $61 \%$ were selected due to their participation in clinical trials. Subsequent studies selected only patients undergoing extrapleural pneumonectomy (EPP) (Kao et al, 2011) or only patients with occupational dust exposure seeking compensation from the Dust Disease Board (Kao et al, 2013). In a fourth study, patients were excluded if they had a history of inflammatory disease, a second primary, active infection or insufficient follow-up data (Pinato et al, 2012). These were therefore also studies with heterogeneous patient populations receiving different treatment approaches.

We set out to validate NLR at diagnosis and at the time of starting treatment, using pre-specified selection criteria and therefore screened all consecutive patients where: (a) the disease under study was confirmed, (b) the variable under study was available and (c) there were no co-existing confounders that may influence the disease or variable under study. It would not be possible to validate the prognostic significance of NLR in patients where the target biomarker was unknown, and we therefore maintain the appropriateness of our selection criteria, and have transparently reported the study denominator. Moreover, all other missing data were described in our report, and the resulting multivariate model was the first published NLR study to include previously established clinical and laboratory prognostic variables. Our data were analysed using rigorous statistical methodology, including the consideration of missing data by multiple imputation.

Kao et al maintain that our data showed 'unusually good overall survival', and this can be adequately refuted by referring to our paper, where we defined overall survival (OS) as being calculated from the time of diagnosis, but also provided OS from the time of commencement of systemic therapy. This was 12.3 months for all patients receiving chemotherapy and 11.7 months for those treated with chemotherapy only (that is, non-surgically). This OS does not differ materially from an OS of 12.1 months for the intervention arm of the landmark cisplatin/ pemetrexed study (Vogelzang et al, 2003) and an OS of 11.7 months for the chemotherapy-naive group of the initial study of NLR in MPM (Kao et al, 2010).

The results were indeed not what we had originally anticipated; nevertheless, we have pursued the scientifically and ethically appropriate course of reporting 'negative' results, which are contradictory to others' findings. Failure to publish negative findings can lead to substantial publication bias in retrospective analyses of data. Our data were subjected to rigorous statistical methodology and we have reported what we found. Furthermore, the NLR cut-off was not data driven but selected a priori on the basis of the correspondents' own published literature. In our discussion, we cautioned against data-driven cut-offs and note that the NLR literature is not consistent in choice of cut-point, which may also have resulted in contradictory findings across studies. Kao et al refer to other manuscripts confirming the prognostic value of NLR that were published during the period in which our manuscript was in submission and in press and that continue to inform scientific debate. At the time of submission we were not privy to these data, which may reflect either an increasing literature on the importance of NLR or alternatively a positive publication bias in retrospective series.

We acknowledge Kao et al as the initiators of this field in mesothelioma and, as previously stated, had anticipated confirming their initial study (Kao et al, 2010) in an additional cohort. That we were not able to do this is not a repudiation of the potential value of NLR, but an invitation for additional rigorous prospective research in the field to fully define the place of this potential prognostic marker.

\section{REFERENCES}

Kao SC, Klebe S, Henderson DW, Reid G, Chatfield M, Armstrong NJ, Yan TD, Vardy J, Clarke S, Van Zandwijk N, Mccaughan B (2011) Low calretinin expression and high neutrophil-to-lymphocyte ratio are poor prognostic factors in patients with malignant mesothelioma undergoing extrapleural pneumonectomy. J Thorac Oncol 6: 1923-1929.

Kao SC, Pavlakis N, Harvie R, Vardy JL, Boyer MJ, Van Zandwijk N, Clarke SJ (2010) High blood neutrophil-to-lymphocyte ratio is an indicator of poor prognosis in malignant mesothelioma patients undergoing systemic therapy Clin Cancer Res 16: 5805-5813.

Kao SC-H, van Zandwijk N, Clarke S (2014) Comment on 'Existing prognostic models, but not neutrophil-to-lymphocyte ratio, are prognostic in malignant mesothelioma'. Br J Cancer 111: 2376.

Kao SC, Vardy J, Chatfield M, Corte P, Pavlakis N, Clarke C, Van Zandwijk N, Clarke S (2013) Validation of prognostic factors in malignant pleural mesothelioma: a retrospective analysis of data from patients seeking compensation from the New South Wales dust diseases board. Clin Lung Cancer 14: 70-77.

Pinato DJ, Mauri FA, Ramakrishnan R, Wahab L, Lloyd T, Sharma R (2012) Inflammation-based prognostic indices in malignant pleural mesothelioma. I Thorac Oncol 7: 587-594.

Vogelzang NJ, Rusthoven JJ, Symanowski J, Denham C, Kaukel E, Ruffie P, Gatzemeier U, Boyer M, Emri S, Manegold C, Niyikiza C, Paoletti P (2003) Phase III study of pemetrexed in combination with cisplatin versus cisplatin alone in patients with malignant pleural mesothelioma. J Clin Oncol 21: 2636-2644.

*Correspondence: Dr TM Meniawy; E-mail: tarek.meniawy@uwa.edu.au Published online 8 April 2014

(c) 2014 Cancer Research UK. All rights reserved 0007-0920/14

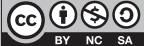

http://creativecommons.org/licenses/by-nc-sa/3.0/

$\mathrm{BIC}$ OPEN 0

\section{Comment on 'Interventions to improve exercise behaviour in sedentary people living with and beyond cancer: a systematic review'}

\section{$\mathrm{J} \mathrm{M} \mathrm{Broderick}^{*}, 1$, J Hussey ${ }^{1}$ and D M O'Donnell ${ }^{2}$}

${ }^{1}$ Department of Physiotherapy, School of Medicine, Trinity Centre for Health Science, St James's Hospital, St James's Street, Dublin 8, Ireland and ${ }^{2}$ Academic Unit of Clinical and Medical Oncology, St James's Hospital, St James's Street, Dublin 8, Ireland

Sir,

We read with great interest the review by Bourke et al (2014). We agree there is a dearth of evidence that any specific intervention results in improved adherence to physical activity guidelines in cancer patients and survivors but would like, respectfully, to offer some further observations.
The authors, in our view, could distinguish more clearly between 'Physical Activity' and 'Exercise' and acknowledge that 'sedentary behaviour' can be independent of physical activity levels. The terms 'exercise behaviour' and 'physical activity' are not interchangeable. 'Physical Activity' refers to body movement produced by the contraction of skeletal muscles and that increases 